\title{
pH Responsiveness of polyelectrolyte dendrimers: a dynamical perspective
}

\author{
Xin Li, ${ }^{a b}$ Michaela Zamponi, ${ }^{b c}$ Kunlun Hong, ${ }^{d}$ Lionel Porcar, ${ }^{e}$ Chwen-Yang Shew, ${ }^{f}$ Timothy Jenkins, ${ }^{g}$ \\ Emily Liu, ${ }^{a}$ Gregory S. Smith, ${ }^{b}$ Kenneth W. Herwig, ${ }^{* b}$ Yun Liu ${ }^{* g h}$ and Wei-Ren Chen*bij
}

\author{
Received 12th July 2010, Accepted 28th October 2010 \\ DOI: $10.1039 / \mathrm{c} 0 \mathrm{sm} 00671 \mathrm{~h}$
}

\begin{abstract}
A combined quasi-elastic neutron scattering (QENS) and high-resolution solution NMR spectroscopy study was conducted to investigate the internal dynamics of aqueous $\left(\mathrm{D}_{2} \mathrm{O}\right)$ G5 PAMAM dendrimer solutions as a function of molecular protonation at room temperature. Localized motion of the dendrimer segments was clearly exhibited in the QENS data analysis while the global, center-of-mass translational diffusion was measured by NMR. Our results unambiguously demonstrate an increased rapidity in local scale $(\sim 3 \AA)$ motion upon increasing the molecular protonation. This is contrary to an intuitive picture that increased charge stiffens the dendrimer segments thereby inhibiting local motion. These charge-induced changes may be a result of interactions with the surrounding counterions and water molecules as the segments explore additional intra-dendrimer volume made available by slight electrostatic swelling and redistribution of mass in the dendrimer interior. This observation is relevant to development of a microscopic picture of dendrimer-based packages as guest-molecule delivery vehicles because reorganization of the confining dendrimer segments must be a precursor to guestmolecule release.
\end{abstract}

\section{Introduction}

Multi-branched dendritic macromolecules have captured the interest of soft matter scientists due to the structural dichotomy arising from their colloidal resemblance and polymeric architecture. ${ }^{1}$ Their unique molecular characteristics, including a well-defined size and the variety of achievable chemical functionalities, have given rise to many industrial applications of technological importance. ${ }^{2,3}$ Extensive computational, experimental and theoretical studies ${ }^{1}$ in the past decades are evidence of the continuing scientific fascination with dendrimers since their first synthesis in the 1980s. ${ }^{4}$

Among this class of synthetic macromolecules, polyamidoamine dendrimers (PAMAM) with ethylenediamine cores have been considered for development as delivery agents for a wide spectrum of guest materials in aqueous environments. ${ }^{3-6}$ For

${ }^{a}$ Department of Mechanical, Aerospace \& Nuclear Engineering, Rensselaer Polytechnic Institute, Troy, NY, 12180, USA

${ }^{b}$ Neutron Scattering Science Division, Oak Ridge National Laboratory, Oak Ridge,TN,37831,USA.E-mail: chenw@ornl.gov; herwigkw@ornl. gov

'Jülich Centre for Neutron Science, Forschungszentrum Jülich, 52425 Jülich, Germany

${ }^{d}$ The Center for Nanophase Materials Sciences, Oak Ridge National Laboratory, Oak Ridge, TN, 37831, USA

${ }^{e}$ Institut Laue-Langevin, B.P. 156, F-38042 Grenoble Cedex 9, France

${ }^{f}$ Department of Chemistry, City University of New York, College of Staten Island, Staten Island, NY, 10314, USA

${ }^{g}$ The NIST Center for Neutron Research, National Institute of Standards and Technology, Gaithersburg, MD, 20899-6100, USA. E-mail: yunliu@ nist.gov

${ }^{h}$ Department of Chemical Engineering, University of Delaware, Newark, DE, 19716, USA

${ }^{i}$ Joint Institute for Neutron Sciences, Oak Ridge National Laboratory, Oak Ridge, TN, 37831, USA

${ }^{j}$ Department of Chemical \& Biomolecular Engineering, the University of Tennessee, Knoxville, TN, 37996-2200, USA example, the merits of this polyelectrolyte dendrimer in targeted drug-delivery applications (where the release of an exogenous drug can be precisely controlled by the stimulus-response mechanism) have been thoroughly exploited. ${ }^{1}$ In such biomedical uses, the efficiency of a dendrimer-based delivery agent is a consequence of its sophisticated architecture and its structural adaptability in dynamically reacting to variations in external thermodynamical conditions.

Extensive computational and experimental efforts have been devoted to investigating the connection between the equilibrium structure of PAMAM dendrimers in solution and their molecular protonation ${ }^{7}$ which can be precisely controlled by adjusting the solution $\mathrm{pH}$. The structural response to charging is manifested as a transition in the intra-molecular density profile where mass is redistributed from the central region to the periphery of the dendrimer along with moderate electrostatic swelling. ${ }^{8-12}$ However, little is known of the effect of charging on the dendrimer dynamics. The confinement of guest molecules (e.g. drugs) and their controlled release must be related to the internal dynamical processes of their host. The structural rearrangement that redistributes mass in response to dendrimer charging is itself a dynamical process resulting in changes to the local environment of the amines and thereby affecting their local motion. Fundamental insight into the internal dynamical processes of a dendrimer under relevant operational conditions are therefore of significant scientific and technical importance. In this study, the internal segmental motions of generation 5 (G5) PAMAM dendrimers dissolved in $\mathrm{D}_{2} \mathrm{O}$ were resolved as a function of molecular charge using high resolution solution NMR measurement and quasi-elastic neutron scattering (QENS). The measured incoherent scattering function shows that the internal segmental dynamics of a dendrimer evolve in response to an increase of the electrostatic interaction. 


\section{Materials}

Biomedical grade amine-terminated poly(amidoamine) dendrimers (PAMAM) (Generation 5) were purchased from Dendritech Inc., Midland, MI, USA. ${ }^{13}$ Purification of the samples used in this work is given elsewhere.$^{8-12}$ Deuterium chloride and deuterium oxide were obtained from Cambridge Isotope Laboratories, Inc., Andover, MA, USA. ${ }^{13}$ The samples studied in this investigation were made by dissolving PAMAM dendrimer in solutions of $\mathrm{DCl}$ and $\mathrm{D}_{2} \mathrm{O}$, and the preparation is detailed in a separate reference. ${ }^{8}$ The solution acidity is represented by $\alpha$, which defines the molar ratio of $\mathrm{DCl}$ to that of the terminal amines. The concentration for both the NMR and QENS measurements was $5 \mathrm{wt} \%$ in high-quality heavy water $\left(\mathrm{D}_{2} \mathrm{O}\right.$, $99.9 \%)$.

\section{Solution NMR measurement}

High resolution solid state NMR has been extensively used to characterized detailed structural characteristics of PAMAM dendrimer systems. ${ }^{14-16}$ The size and dynamics of IAPP peptide species is also investigated by diffusion NMR spectroscopy. ${ }^{17} \mathrm{In}$ our work, it was used to explore the dependence of the diffusional motion of PAMAM dendrimer in aqueous solution on the molecular charge. NMR experiments were carried out using a Bruker Avance NMR spectrometer operating at $400 \mathrm{MHz}$. The instrument was equipped with a gradient amplifier. One-dimensional ${ }^{1} \mathrm{H}$ experiments were performed using standard pulse sequences from the Bruker library. ${ }^{18}$ Diffusion experiments were carried out using pulsed field-gradient spin echo NMR (PFGSE NMR) with 16 increments in the gradient strength (2-95\%). The gradient coil strength was calibrated with diffusion of HDO in $\mathrm{D}_{2} \mathrm{O}\left(D=1.91 \times 10^{-9} \mathrm{~m}^{2} / \mathrm{s}\right.$ at $\left.297.5 \mathrm{~K}\right)$. The diffusion coefficients $(D)$ were extracted from the slope of the intensity decays of spinecho signal using the Stejskal-Tanner equation: ${ }^{19,20}$

$$
I / I(0)=\exp \left[-D \gamma^{2} g^{2} \delta^{2}(\Delta-\delta / 3)\right]
$$

where $I$ and $I(0)$ are intensities of the echo signal with and without the magnetic field gradient, respectively, $\Delta$ the time between the gradient pulses (diffusion time), $\gamma$ the gyromagnetic ratio, $g$ the gradient strength, $\delta$ the duration of gradient and $D$ the diffusion coefficient. The peak in the spectrum at $3.65 \mathrm{ppm}$ was used to calculate the diffusion coefficient.

\section{Quasi-elastic neutron scattering measurement}

The experiments were carried out using the high-resolution backscattering spectrometer BaSiS at the Spallation Neutron Source (SNS) in Oak Ridge National Lab (ORNL). BaSiS features an elastic energy resolution of $3.4 \mu \mathrm{eV}$ (FWHM) and the dynamical range of energy transfer, $\omega$, was selected as \pm 100 $\mu \mathrm{eV}{ }^{21}$

The samples were confined to a $0.5 \mathrm{~mm}$ annulus in a cylindrical niobium sample cell in order to minimize multiple scattering. All samples, including a vanadium standard used as the instrument resolution function and pure solvent in the niobium cell used as a background, were measured at seven values of momentum transfer, $Q$, from $0.3 \AA^{-1}$ to $1.5 \AA^{-1}$ at room temperature $(300 \mathrm{~K})$. Based on previous results from small angle scattering (SANS), there could be considerable coherent scattering at the lowest $Q$ value, consequently this spectrum has been suppressed in the analysis. ${ }^{22}$ The large incoherent cross-section of the hydrogen atom means that the measured signals are dominated by contributions from the hydrogen atoms in the amine groups of the dendrimer segments. The solvent and niobium sample cell contributions were subtracted from the data sets leaving only the signal from the dendrimer. No correction for multiple scattering was made because the transmission was at the level of $87 \%$.

\section{Model of the incoherent scattering function}

The incoherent scattering function can be considered as the convolution of the dendrimer global, center-of-mass translational Brownian motion with its internal local motion ${ }^{23}$

$$
S_{\mathrm{inc}}(Q, \omega)=S_{\mathrm{int}}(Q, \omega) \otimes S_{\mathrm{trans}}(Q, \omega)+B(Q, \omega)
$$

where $S_{\text {inc }}(Q, \omega)$ is the total incoherent scattering function, $S_{\text {int }}(Q, \omega)$ is the contribution from internal motion, and $S_{\text {trans }}(Q, \omega)$ represents the center-of-mass translational diffusion. $B(Q, \omega)$ is an $\omega$-dependent linear background. $S_{\text {trans }}(Q, \omega)$ can be described by a Lorentzian $L_{\text {trans }}(Q, \omega)$ function with half-width at half-maximum (HWHM) of $\Gamma_{\text {trans }}=D_{s} Q^{2}$. In this analysis, the self-diffusion coefficient $D_{s}$ was constrained to that obtained from the NMR measurement. In the simplest phenomenological approach, $S_{\text {int }}(Q, \omega)$ may be represented as the sum of an elastic term and a quasi-elastic, Lorentzian contribution with HWHM, $\Gamma_{\text {internal. }} A_{0}(Q)$ describes the distribution of intensity between the two terms and the Debye-Waller factor accounts for intensity loss due to vibrational motion:

$$
S_{\text {int }}(Q, \omega)=e^{-Q^{m}\left\langle u^{2}\right\rangle / 3}\left[A_{0}(Q) \delta(\omega)+\left(1-A_{0}(Q)\right) L_{\text {int }}(Q, \omega)\right]
$$

Finally, the measured intensity is represented as

$$
\begin{gathered}
S_{\mathrm{inc}}(Q, \omega)=\left\{e^{-Q_{w}\left\langle u^{2}\right\rangle / 3}\left[A_{0}(Q) \delta(\omega)+\left(1-A_{0}(Q)\right) L_{\mathrm{int}}(Q, \omega)\right]\right. \\
\left.\otimes L_{\text {trans }}(Q, \omega)+B(Q, \omega)\right\} \otimes R(Q, \omega)
\end{gathered}
$$

where $R(Q, \omega)$ is the resolution function of the instrument. It is important to note that a recent MD simulation ${ }^{24}$ suggests that the characteristic relaxation time corresponding to overall rotationally diffusive motion of dendrimer molecule is much longer than the accessible temporal range of the spectrometer. Therefore, in our QENS data analysis this dynamical contribution has been neglected.

\section{Results and discussion}

Fig. 1 gives the linear Stejskal-Tanner plots obtained from solutions of G5 PAMAM. The single-exponential decay in the NMR amplitude indicates that the samples consist predominantly of individual dendrimer molecules. Upon increasing $\alpha$, the extracted self-translational diffusion coefficient $D_{s}$ is seen to first decrease when the primary amines are charged $(\alpha=1)$ and then slightly increase when dendrimer is fully charged $(\alpha=2)$. In comparison to its non-charged state, SANS experiments show that a G5 PAMAM dendrimer exhibits only minor swelling in 


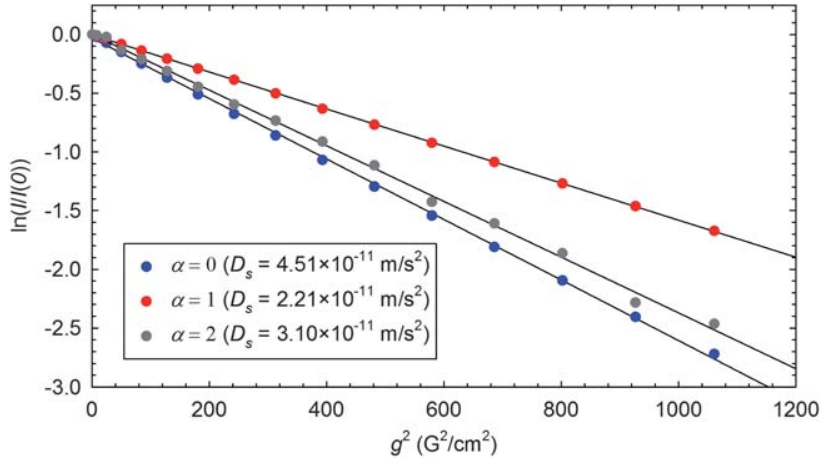

Fig. 1 The Stejskal-Tanner plot of G5 PAMAM dendrimer at three different levels of molecular protonation. The extracted self diffusion coefficients $D_{s}$ are given in the legends.

global size ( $5 \%$ increase in radius of gyration $R_{G}$ ) when it is fully charged in $\mathrm{D}_{2} \mathrm{O} .{ }^{9}$ This non-trivial functional dependence of $D_{s}$ on $\alpha$ cannot be attributed only to the change in hydrodynamic radius of the dendrimer via the Stokes-Einstein formula. Varying surface conditions due to progressive charging and enhanced counterion condensation could affect $D_{s}$ more strongly than the slight variation in physical size.

An example of the QENS spectra is presented in Fig. 2 where the model fitting was performed over the energy range $\pm 100 \mu \mathrm{eV}$ at scattering wavevector $Q=0.9 \AA^{-1}$. The quasi-elastic broadening due to internal motion of the dendrimer (dashed line) is clearly seen after separating the contribution from the global translational diffusion component (shaded area) determined from the NMR measurement. In light of the complexity arising from its hierarchical structure, which inherently gives rise to a dynamical behavior characterized by multi-step relaxations spanning a temporal range of several orders of magnitude, a complete dynamical picture of the dendrimer will not be fully described by our simplified, single-Lorentzian model nor captured by our measurements. However, the nanosecond and faster time range seems to be well-modeled by a single relaxation process as defined in eqn (4) and this is the relevant time scale for describing the dynamics of a typical guest drug molecule hosted in PAMAM dendrimer. ${ }^{25}$

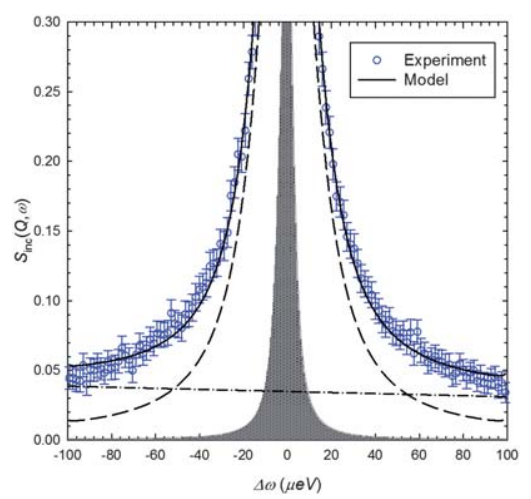

Fig. 2 QENS intensity (blue symbols) recorded at $Q=0.9 \AA^{-1}$, the total fitted curve (solid line), the corresponding components of translational diffusion (shaded area), the Lorentzian (dashed line) and background (dash-dotted line).
The QENS spectra are presented in Fig. 3 at three levels of molecular charging. Qualitatively, within the studied $Q$ and dynamical range, $S_{\mathrm{inc}}(Q, \omega)$ broadens (faster motions) in response to an increase in molecular protonation. As shown in Fig. 3, good agreement between the model of eqn (4) and experiment was found within the entire $Q$ range. The continuous increase of $\Gamma_{\text {internal }}(Q)$ with $Q$ and its non-zero value at $Q=0$ (Fig. 4a) demonstrate that the internal dendrimer dynamics can be phenomenologically well characterized by a localized diffusive motion which becomes more rapid as $\alpha$ increases. This surprising observation is in contradiction to an intuitive expectation that charging the dendrimer would make it less flexible and inhibit local diffusive motion of the segments. The answer may lie in consideration of the relevant length scales. Significant rearrangement of dendrimer segments likely occurs on much longer length scales (and time scales) than sampled in the present experiment. Care is needed in interpreting the dynamical picture on length scales greater than $\sim 9 \AA\left(Q^{2}<0.5 \AA^{-2}\right)$ since the data analysis is complicated by several unaccounted factors such as the hydrodynamic interaction, ${ }^{26}$ intra-dendrimer collective motion $^{27,28}$ and rotational motion of the segments. ${ }^{29}$ This evolution in dynamics can also be visualized by the self intermediate scattering functions $S_{\text {inc }}(Q, t)$ shown in Fig. 4b-d calculated from the fits to eqn (4). As $\alpha$ increases, a more rapid decay of $S_{\text {inc }}(Q, t)$ is observed.

Additional insight, particularly into the relevant length scale of the motion, can be obtained by applying a simple model of localized diffusion which has been successfully applied in the analysis of alkyl chain dynamics ${ }^{30,31}$ where the hydrogen atoms are assumed to be moving within an impenetrable spherical volume. A diffusion coefficient $D$ and the sphere radius $R$ are used to describe this confined diffusive motion. The fitting curves and results are shown in Fig. 5 and 6. Despite fluctuations, the tendency of the internal diffusion coefficient $D$ also demonstrates that the local motion is faster when the dendrimer is charged. With the increase of dendrimer protonation, the apparent diffusion coefficient is seen to increase steadily: $(1.60 \pm 0.03) \times$ $10^{-10} \mathrm{~m}^{2} / \mathrm{s}(\alpha=0),(2.58 \pm 0.03) \times 10^{-10} \mathrm{~m}^{2} / \mathrm{s}(\alpha=1),(3.11 \pm$ $0.04) \times 10^{-10} \mathrm{~m}^{2} / \mathrm{s}(\alpha=2)$.

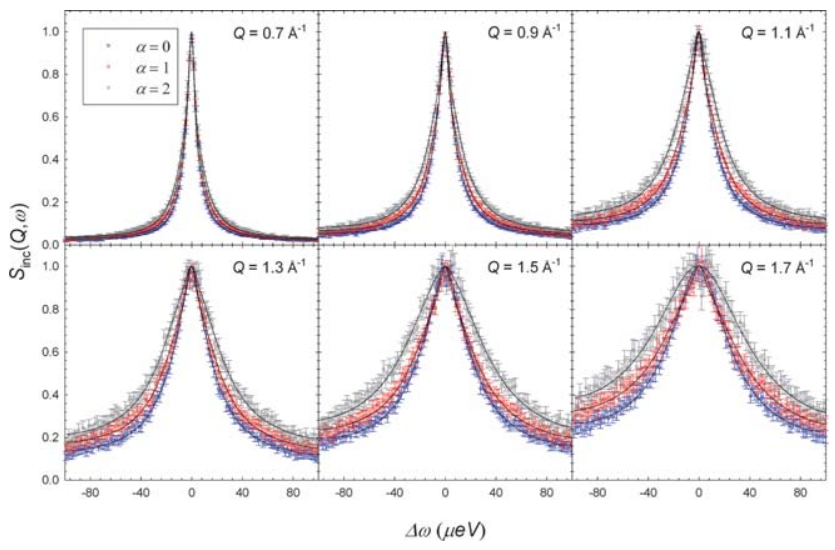

Fig. 3 The incoherent scattering function $S_{\text {inc }}(Q, \omega)$ obtained from G5 PAMAM dendrimer solution with the concentration of $5 \%$ weight and the $\alpha$ values of 0,1 , and 2 . Solid lines correspond to the fitting curves with eqn (4). 

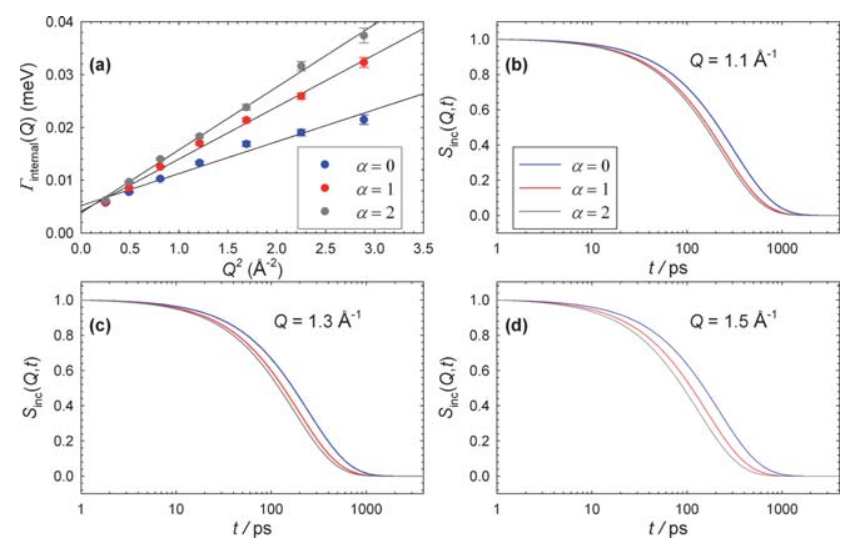

Fig. 4 (a) Evolution of $\Gamma_{\text {internal }}(Q)$ as a function of $Q^{2}$ at three different $\alpha$ values. The corresponding $S_{\text {inc }}(Q, t)$ obtained at $Q=1.1 \AA^{-1}$ (b), $Q=1.3 \AA^{-1}$ (c) and $Q=1.5 \AA^{-1}$ (d).

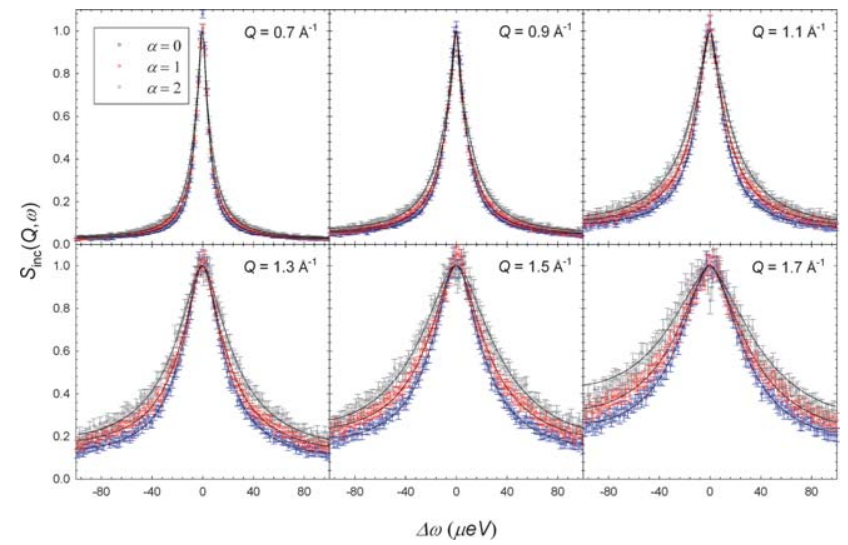

Fig. 5 The incoherent scattering function $S_{\text {inc }}(Q, \omega)$ fitted with the model of the diffusion in a sphere. Solid lines are the fitting curves. The experiment data are the same as in Fig. 3.
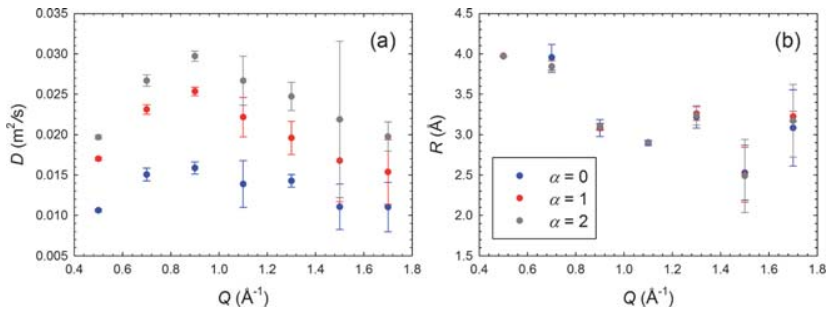

Fig. 6 The result of the QENS data analysis with the diffusion-in-asphere model described in ref. 26 and 27: (a) the diffusion coefficient, (b) the radius of the sphere.

Our observation is contrary to a coarse-grained Brownian dynamics (BD) simulation which predicts progressively more sluggish internal motion with an increase in the intra-dendrimer electrostatic interaction. ${ }^{32}$ However, whether this mean-field approach can accurately depict microscopic dynamical information of a dendrimer, remains a critical open question. This disagreement between the present experiment and coarse-grained simulation reflects the importance of incorporating all the complexity of the real system including the various interactions involving charged amines, counterions and water molecules explicitly, particularly at these relatively short length scales $(<10 \AA)$.

The sphere radius describing the extent of the motion is about $2.8 \AA$ and $\alpha$-independent. This interesting behavior indicates that on the local length scale, charging the dendrimer weakens the average interactions experienced by the amine groups. This could arise due to some combination of an increasing degree of counterion condensation which progressively modulates the electrostatic interaction, ${ }^{33}$ the varying strength of hydrogen bonding due to penetrating water, ${ }^{33}$ and the additional intra-dendrimer space created by conformational change ${ }^{8-12,34-36}$ which, to some extent, relieves local steric crowding hindering the segmental maneuverability. The further implication is that the range of motion of the amine hydrogen atoms is limited on the time scale of this measurement $(\approx 1 \mathrm{~ns})$ which implies that larger amplitude motions involving significant rearrangement of the dendrimer backbone occur on a much slower time scale.

\section{Conclusions}

In conclusion, we provide the first conclusive experimental evidence demonstrating clear dependence of the internal dynamics of G5 PAMAM dendrimers in $\mathrm{D}_{2} \mathrm{O}$ on their molecular charge. We believe this enhancement of local scale dynamics of the dendrimer segments is closely related to their interactions with counterions and water molecules, as well as the availability of intra-dendrimer volume allowing additional maneuverability. This charge-induced dynamical change may provide critical information for general polyelectrolyte dendrimer-based packages used as delivery vehicles under operational conditions with $\mathrm{pH}$ values of biological relevance.

\section{Acknowledgements}

This research at Oak Ridge National Laboratory's SNS was sponsored by the Scientific User Facilities Division, Office of Basic Energy Sciences, U.S. Department of Energy. CNMS is sponsored at ORNL by the Division of Scientific User Facilities, U.S. Department of Energy.

\section{Notes and references}

1 M. Ballauff and C. N. Likos, Angew. Chem., Int. Ed., 2004, 43, 2998. 2 B. Voit, J. Polym. Sci., Part A: Polym. Chem., 2000, 38, 2505.

3 M. Fisher and F. Vögtle, Angew. Chem., Int. Ed., 1999, 38, 884.

4 D. A. Tomalia, H. Baker, J. Dewald, M. Hall, G. Kallos, S. Martin, J. Roeck, J. Ryder and P. Smith, Polym. J., 1985, 17, 117.

5 R. Esfand and D. A. Tomalia, Drug Discovery Today, 2001, 6, 427.

6 U. Boas and P. M. H. Heegaard, Chem. Soc. Rev., 2004, 33, 43.

7 SeeA. W. Opitz and N. J. Wagner, J. Polym. Sci., Part B: Polym. Phys., 2006, 44, 3062 and references therein.

8 W.-R. Chen, L. Porcar, Y. Liu, P. D. Butler and L. J. Magid, Macromolecules, 2007, 40, 5887.

9 L. Porcar, Y. Liu, R. Verduzco, K. Hong, P. D. Butler, L. J. Magid, G. S. Smith and W.-R. Chen, J. Phys. Chem. B, 2008, 112, 14772.

10 L. Porcar, K. Hong, P. D. Butler, K. W. Herwig, G. S. Smith, Y. Liu and W.-R. Chen, J. Phys. Chem. B, 2010, 114, 1751.

11 Y. Liu, L. Porcar, K. Hong, C.-Y. Shew, X. Li, E. Liu, P. D. Butler, K. W. Herwig, G. S. Smith and W.-R. Chen, J. Chem. Phys., 2010, 132, 124901.

12 Y. Liu, C.-Y. Chen, H.-L. Chen, K. Hong, C.-Y. Shew, X. Li, L. Liu, Y. B. Melnichenko, G. S. Smith, K. W. Herwig, L. Porcar and W.-R. Chen, J. Phys. Chem. Lett., 2010, 1, 2020. 
13 In their own activities as scientific institutions, ORNL uses many different materials, products, types of equipment, and services. However, ORNL does not approve, recommend, or endorse any product or proprietary material.

14 P. E. S. Smith, J. R. Brender, U. H. N. Dürr, J. Xu, D. G. Mullen, M. M. Banaszak Holl and A. Ramamoorthy, J. Am. Chem. Soc., 2010, 132, 8087.

15 A. Mecke, D.-K. Lee, A. Ramamoorthy, B. G. Orr and M. M. Banaszak Holl, Langmuir, 2005, 21, 8588.

16 A. Mecke, S. Uppuluri, T. M. Sassanella, D.-K. Lee, A. Ramamoorthy, J. R. Baker Jr., B. G. Orr and M. M. Banaszak Holl, Chem. Phys. Lipids, 2004, 132, 3.

17 R. Soong, J. R. Brender, P. M. Macdonald and A. Ramamoorthy, J. Am. Chem. Soc., 2009, 131, 7079.

18 Detailed information can be found at the following website: http:// www.bmrb.wisc.edu/pulse_seq/spec_bruker.html.

19 E. O. Stejskal and J. E. Tanner, J. Chem. Phys., 1965, 42, 288.

20 E. O. Stejskal, J. Chem. Phys., 1965, 43, 3597.

$21 \mathrm{http}: / /$ neutrons.ornl.gov/instruments/SNS/BASIS/.

22 J. Pérez, J.-M. Zanotti and D. Durand, Biophys. J., 1999, 77, 454.

23 J.-M. Zanotti, M.-C. Bellissent-Funel and J. Parello, Biophys. J., 2002, 83, 2792.
24 J. Aumanen, T. Kesti, V. Sundstorm, G. Teobaldi, F. Zerbetto, N. Werner, G. Richardt, J. Heyst, F. Vögtle and J. KorppiTommola, J. Phys. Chem. B, 2010, 114, 1548.

25 I. Tanis and K. Karatasos, J. Phys. Chem. B, 2009, 113, 10984.

26 G. Nägele, Phys. Rep., 1996, 272, 215.

27 B. Stark, C. Lach, B. Farago, H. Frey, C. Schlenk and B. Stühn, Colloid Polym. Sci., 2003, 281, 593.

28 S. Rathgeber, M. Monkenbusch, M. Kreitschmann, V. Urban and A. Brulet, J. Chem. Phys., 2006, 125, 204908.

29 P. K. Maiti and W. A. Goddard, J. Phys. Chem. B, 2006, 110, 25628.

30 F. Volino and A. J. Dianoux, Mol. Phys., 1980, 41, 271.

31 L. Carpentier, M. Bée, A. M. Giroud-Godquin, P. Maldivi and J. C. Marchon, Mol. Phys., 1989, 68, 1367.

32 S. V. Lyulin, A. A. Darinskii, A. V. Lyulin and M. A. J. Michels, Macromolecules, 2004, 37, 4676.

33 Y. Niu, L. Sun and R. M. Crooks, Macromolecules, 2003, 36, 5725.

34 R. Blaak, S. Lehmann and C. N. Likos, Macromolecules, 2008, 41, 4452 .

35 Y. Liu, V. S. Bryantsev, M. S. Diallo and W. A. Goddard III, J. Am. Chem. Soc., 2009, 131, 2798.

36 W. Tian and Y. Ma, J. Phys. Chem. B, 2009, 113, 13161. 